\title{
Urinary tract infection of rare pathogen Raoultella planticola
}

Taylor Bullock ${ }^{1 *}$, Robert Cain ${ }^{1}$

${ }^{1}$ Cleveland Clinic Lerner College of Medicine, Cleveland, OH USA

*Corresponding Author: Taylor Bullock, Cleveland Clinic Lerner College of Medicine 9500 Euclid Avenue, Cleveland, OH 44195, USA

Email: bulloct2@ccf.org

Received Date: July 29, 2020; Accepted Date: July 05, 2020; Published Date: Augusł 11, 2020.

Citation: Taylor Bullock, Robert Cain (2020), Urinary tract infection of rare pathogen Raoultella planticola. J Clinical Case Reports and Studies, 1(3): Doi:10.31579/2690-8808/021

Copyright: ( ) 2020. Taylor Bullock. This is an open-access article distributed under the terms of the Creative Commons Attribution License, which permits unrestricted use, distribution, and reproduction in any medium, provided the original author and source are credited.

\begin{abstract}
Raoultella planticola is a gram-negative bacillus that is rarely associated with clinical infections. We present a patient with recurrent urinary tract infections and glioblastoma multiforme that grew Raoultella planticola with urine culture. Of the 32 documented cases to date (only 8 of them in the United States), this is one of the few cases where the Raoultella planticola infection was a UTI in a patient with recurrent cystitis $[1,2]$. The patient was successfully treated with cephalexin and nitrofurantoin.

Keywords: UTI; Raoultella planticola
\end{abstract}

\section{Introduction}

Raoultella planticola is a gram-negative, non-motile, aerobic bacillus that is associated with water, soil, and aquatic environments [3]. It was originally classified as a member of the genus Klebsiella, before reclassified as Raoultella spp. Invasive infection from Raoultella was first reported in 1984 in a patient with sepsis in France. This bacillus can be found in scombroid fish and is able to produce histamine [4]. Current literature suggests that Raoultella planticola is a rare cause of human infections in the clinic $^{5}$.

\section{Case Presentation}

The nurse of a 78-year-old male patient called the hospital and reported that the patient's urine was dark, cloudy, and foul smelling. The patient was using an external catheter. The patient had a past medical history of recurrent UTIs, hypertension, coronary artery disease with a myocardial infarction, and glioblastoma multiforme of the brain. His recurrent UTIs started 7 months prior to presentation. His symptoms included increased urinary frequency, pain and pressure with urination, and urine that was cloudy and dark. He had been treated with a 7-day course of ciprofloxacin and a 7-day course of sulfamethoxazole-trimethoprim with minimal reduction in urinary symptoms. He was diagnosed with glioblastoma multiforme five years prior, after experiencing a simple partial seizure with left hemibody numbness and weakness, along with slurred speech. His symptoms were reported to of lasted approximately an hour. The glioblastoma was located in the frontotemporal lobe and was IDH1 negative with EGFR not amplified. The mass was resected and the patient was treated with radiation and 12 cycles of temozolomide. The patient's glioblastoma recurred two years later in the right parietal lobe and was treated with stereotactic radiosurgery (gamma knife). Within the last year, more tumor growth had been resected and the patient was treated with intraparenchymal topotecan by Convection-Enhanced Delivery in a clinical trial.

A urine culture was ordered, which grew greater than 100,000 colonyforming units of Escherichia coli and greater than 100,000 colonyforming units of Raoultella planticola per milliliter. The Escherichia coli was resistant to ampicillin, ciprofloxacin, and levofloxacin. The Raoultella planticola was resistant to ampicillin, but was sensitive to trimethoprim/sulfamethoxazole, amoxicillin/clavulanic acid, ampicillin/sulbactam, cefazolin, cefepime, ceftazidime, ceftriaxone, cefuroxime, ciprofloxacin, ertapenem, gentamicin, levofloxacin, nitrofurantoin, and tobramycin (see Table 1). 


\begin{tabular}{|l|c|}
\hline Antibiotic & Minimal Inhibitory Concentration $(\boldsymbol{\mu g} / \mathbf{m l})$ \\
\hline Amoxicillin/Clavulanic Acid & $\leq 2$ \\
\hline Ampicillin & $\geq 32$ \\
\hline Ampicillin/Sulbactam & 4 \\
\hline Cefazolin & $\leq 4$ \\
\hline Cefepime & $\leq 1$ \\
\hline Ceftazidime & $\leq 1$ \\
\hline Ceftriaxone & $\leq 1$ \\
\hline Cefuroxime & $\leq 1$ \\
\hline Ciprofloxacin & $\leq 0.25$ \\
\hline Ertapenem & $\leq 0.5$ \\
\hline Gentamicin & $\leq 1$ \\
\hline Levofloxacin & $\leq 0.12$ \\
\hline Nitrofurantoin & $\leq 16$ \\
\hline Tobramycin & $\leq 1$ \\
\hline Trimeth/Sulfa & $\leq 20$ \\
\hline
\end{tabular}

Table 1: In vitro susceptibility profile.

The patient was treated with a 7-day course of cephalexin, followed by prophylactic nitrofurantoin for 90 days. The patient reported cessation of urinary symptoms. Three months later, the patient was still experiencing urinary symptoms. The wife said that patient had noticed a small blood clot in his urine the day before and felt pressure and pain when he tried to urinate. Urine culture 3 months after no longer showed the presence of Raoultella planticola.

\section{Discussion}

Raoultella planticola is a gram-negative aerobic bacillus that is rarely associated with clinical infection. It is typically found in water, soil, and aquatic environments. Of the reported Raoultella planticola infections, the majority were associated with immunocompromised states or malignancy [6]. This case supports the trend of infection with Raoultella planticola occurring in a patient with a malignancy.

Klebsiella spp. have been shown to have a close resemblance with Raoultella planticola. These species share many of the same virulence factors, which suggests that they may have similar courses of human infection, antibiotic sensitivities, as well as developed antibiotic resistances [7]. Because of the ability of Raoultella planticola to acquire antibiotic-resistant plasmid genes, like Klebsiella spp., Raoultella planticola has the potential to become a potential health threat in the future $[5,8]$. In this patient, Raoultella planticola was successfully eliminated with cephalexin and nitrofurantoin.

\section{References}

1. Skelton IV WP, Taylor Z, Hsu J. (2017). A rare case of Raoultella planticola urinary tract infection in an immunocompromised patient with multiple myeloma. ID Cases. 8:9-11.

2. Fager C, Yurteri-Kaplan L. (2018). Urinary tract infection with rare pathogen Raoultella Planticola: A post-operative case and review. Urol case reports. 22:76-79.

3. Teo I, Wild J, Ray S, Chadwick D. (2012). A Rare Case of Cholecystitis Caused by Raoultella planticola. Case Rep Med. 2012:601641.

4. Kanki M, Yoda T, Tsukamoto T, Shibata T. (2002). Klebsiella pneumoniae Produces No Histamine: Raoultella planticola and Raoultella ornithinolytica Strains Are Histamine Producers. Appl Environ Microbiol. 68(7):3462-3466.

5. Cho YJ, Jung EJ, Seong JS, et al. (2016). A Case of Pneumonia Caused by Raoultella planticola. Tuberc Respir Dis (Seoul). 79(1):42-45. doi:10.4046/trd.2016.79.1.42

6. Chun S, Yun JW, Huh HJ, Lee NY. (2014). Low virulence? Clinical characteristics of Raoultella planticola bacteremia. Infection. 42(5):899-904.

7. Podschun R, Fischer A, Ullman U. (2000). Expression of putative virulence factors by clinical isolates of Klebsiella planticola. J Med Microbiol. 49(2):115-119.

8. Castanheira M, Deshpande LM, DiPersio JR, Kang J, Weinstein MP, Jones RN. (2009). First descriptions of blaKPC in Raoultella spp. (R. planticola and R. ornithinolytica): report from the SENTRY Antimicrobial Surveillance Program. J Clin Microbiol. 47(12):4129-4130. 